\title{
Acoustic waves in granular materials
}

\author{
O. Mouraille, S. Luding \\ Particle Technology, Nano-Structured Materials, DelftChemTech, Delft University of Technology, Julianalaan 136, 2628BL Delft, Netherlands \\ O.J.P.Mouraille@tudelft.nl, S.Luding@tudelft.nl
}

\begin{abstract}
Dynamic simulations with discrete elements are used to obtain more insight into the wave propagation in dense granular media. A small perturbation is created on one side of a dense, static packing and examined during its propagation until it arrives at the opposite side. The influence of polydispersity is studied by randomly varying the particle sizes by a tiny amount. A size variation comparable to (or larger than) the typical contact deformation, considerably changes sound propagation, i.e., the transmission spectrum becomes discontinuous and lower frequencies are transmitted better in the polydisperse packing. The inter-particle friction affects the dispersion relation, it increases the propagation speed and leads to an extended linear, large wavelength regime.
\end{abstract}

Key words: DEM simulation, granular matter, sound propagation, micro-macro theory, friction, rotation

\section{A. Introduction}

The sound propagation mechanisms inside dense granular matter are challenging the attempts to describe them because of the discrete, inhomogeneous nature of the material [1-4]. Phenomena like dissipation, scattering, and dispersion are difficult to predict based on the material state and properties. A continuum description of granular materials is generally needed in field application (like oil recovery) because of the huge size of the systems considered. Hence starting from simulations at the particle level, which are necessary to get some insight into the role of micro-parameters, one has to consider a "micro-macro" transition for applications [3]. The ultimate goal is to provide micro-based macroscopic constitutive relations, superior to the more empirical approach.

The use of 3D discrete element method (DEM) simulations allows the study of the role of microparameters such as damping and inter-particle friction. Also the influence of the geometrical (and hence contact) disorder can be examined. In the following, (almost) regular structured packings are studied with weak polydispersity and friction active.

In section B, DEM - also called MD (Molecular Dynamics) - is introduced as well as the granular packing structure. In section $\mathrm{C}$, the wave speeds (for different packings) as obtained from simulation and theory are compared and the frequency content of the waves is examined for three different cases: (i) a regular packing as reference, (ii) a weakly polydisperse packing, and (iii) the latter polydisperse case with friction.

\section{B. Simulation setup}

\section{B.1. DEM Model}

The elementary units of granular materials are mesoscopic grains, which deform under the stress developing at their contacts. Since realistic modeling of the internal deformation of the particles is much too complicated, we relate the normal interaction force to the overlap $\delta$ of two spherical particles. If the sum of all forces, $f_{\mathrm{i}}$, acting on particle $i$, either from other particles, from boundaries or from external forces, is known, the problem is reduced to the integration of Newton's equations of motion for the translational and rotational degrees of freedom:

$$
m_{i} \frac{d^{2}}{d t^{2}} r_{i}=f_{i}, \quad \text { and } \quad I_{i} \frac{d^{2}}{d t^{2}} \varphi_{i}=t_{i},
$$

with the mass $m_{\mathrm{i}}$ of particle $i$, its position $r_{\mathrm{i}}$, its moment of inertia $I_{\mathrm{i}}$, its angular velocity $\omega_{\mathrm{i}}=\mathrm{d} \varphi_{\mathrm{i}} / \mathrm{dt}$ and the total torque $t_{\mathrm{i}}$. Note that the above equation is only valid for isotropic bodies, like spheres as used in this study. The force acting on particle $i$ from particle $j$ can be decomposed into a normal and a tangential part; for a detailed description of the model is given in Ref. [5]. For the results presented in this article we used a simple linear spring model: $|f|=k \delta$, with $k\left(=10^{5} \mathrm{~N} / \mathrm{m}\right)$ the contact stiffness.

\section{B.2. Particle packing}

The configuration considered here is a dense, static packing of grains, with radii $a_{0}(=0.001 \mathrm{~m})$, arranged in a Face Centered Cubic (FCC) structure (density $\approx 0.74$ ), where square layers in the $\mathrm{x}-\mathrm{y}$ plane $(4 \mathrm{x} 4$ particles) are stacked in the $\mathrm{z}$-direction (200 layers).

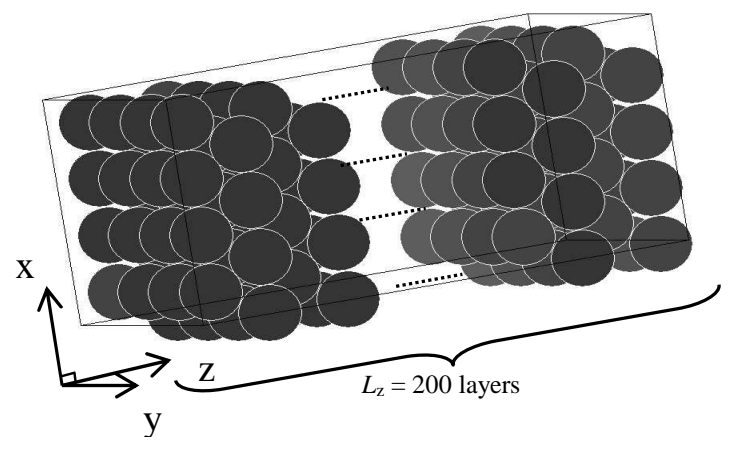

Fig. 1. FCC-structured packing, thin (periodic) in the $x-y$ directions, and elongated (non-periodic) in the z-direction. 
The obtained system is thus thin and elongated in the z-direction where each particle has four contacts inside each square-layer, and eight with particles in both neighbouring layers, corresponding to a coordination number $C=12$.

Polydisperse packings are obtained by introducing a tiny size distribution to the system. This distribution is homogeneous, centered at the original monodisperse value of the radius and has a width $2(\Delta a)$, of the order of the overlap $\delta\left(\delta / a=10^{-3}\right)$. More precisely we studied three different cases with $(\Delta a)=\delta / 2, \delta$ and $2 \delta$. As consequence of this small polydispersity the structured system becomes unstable: A relaxation-simulation is needed to equilibrate the system after the particles sizes are changed. The polydisperse system obtained after relaxation conserves its original FCC structure at the grain scale, since the radius variation is much smaller than $a(\sim 3$ orders of magnitude), however some differences are observed at the contact level. Indeed, a radius variation of $(\Delta a)=2 \delta$ represents a small change of $0.2 \%$ at the grain level but a large change of $200 \%$ at the contact level. This has a direct influence on the coordination numbers which drop down from $C=12$ to $C=11.99,11.22$ and 9.97 for the three cases $(\Delta a)=\delta / 2, \delta$ and $2 \delta$ respectively. For the last case this means a non-negligible reduction of about $17 \%$ of the total amount of contacts of the monodisperse system. Since the effect of this "tiny" radius variation is strong, polydispersity has to be related to the overlap rather than to the particle size, as reported in Ref. [9].

\section{Results}

An initial velocity in the $\mathrm{z}$-direction $\mathrm{v}_{\mathrm{z}}=\mathrm{v}_{0}$ is given to all the particles contained in the first $x$-y-layer $\left(z=z_{0}\right.$ smallest) of the packing, for the other particles in the system the initial velocity is zero $\left(\mathrm{v}_{\mathrm{z}}=0\right.$ if $\left.\mathrm{z} \neq \mathrm{z}_{0}\right)$, see Fig. 2 . This excitation creates a plane compressive $(\mathrm{P})$ wave that propagates through the system along the z-direction. In the following, the 'stress versus time' signals recorded at each layer are analyzed for each of the four systems described in section 2.1. Note that the boundary condition and the excitation method, both have an influence on the stress-time signal shape (data not shown).

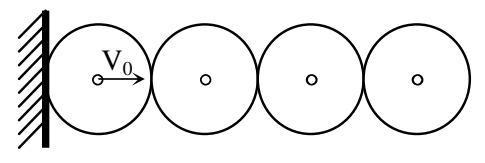

Fig. 2. One-dimensional equivalent schema of the initial excitation method, where the $\mathrm{z}$-direction is horizontal.

\section{C.1. Wave velocities}

One way to define the wave speed is to measure the time it takes the peak of the first pulse to travel a certain distance. Fig. 3 shows two stress-time signals for the monodisperse and a polydisperse, $(\Delta \mathrm{a})=2 \delta$, packing, both recorded at the same distance, 10 layers, from the source - as discussed in detail below. The maxima (first peak) of stress in a space-time plot (data not shown) gives an almost straight line [5]. Just assuming a constant speed, thus disregarding the acceleration of the pulse at the very beginning of the system, see Ref. [5], the slope of this line then gives the speed $v_{p}$ during propagation, see Table 1 in the row labeled "simulation". Note that other points could have been chosen as reference, like $10 \%$ of the first pulse for example. Due to dispersion this gives different values for $\mathrm{v}_{\mathrm{p}}$, but still very close to those presented here, as it is also reported in Ref. [1].

\begin{tabular}{|c|c|c|c|c|}
\hline$(\Delta a)$ & 0 & $\delta / 2$ & $\Delta$ & $2 \delta$ \\
\hline $\mathrm{V}_{\mathrm{pz}}$ (Theory) & 218.4 & 218.4 & 211.6 & 199.7 \\
\hline $\mathrm{v}_{\mathrm{pz}}$ (Simulation) & 211.4 & 211.2 & 205.5 & 183.8 \\
\hline
\end{tabular}

Tab. 1. Comparison of the wave velocities $\left(\mathrm{v}_{\mathrm{pz}}\right.$ given in $\left.\mathrm{m} / \mathrm{s}\right)$ obtained by theoretical prediction with those obtained from simulations.

The simulation results can now be compared with the wave speed predicted from the static material stiffness, if the system is considered as a continuous medium. The stiffness tensor $\boldsymbol{C}$ of the material can be derived from the potential energy density via virtual displacement, see [3], and can be written (in index notation) as:

$$
C_{\alpha \beta \gamma \phi}=\frac{1}{V} \sum_{p \in V}\left(k \sum_{c=1}^{c}\left(l^{2} / 2\right) n_{\alpha}^{c} n_{\beta}^{c} n_{\gamma}^{c} n_{\phi}^{c}\right),
$$

with a representative volume $V$, the contact stiffness $k$, the branch vector $l$ and the normal vectors at the contact $\boldsymbol{n}^{\mathrm{c}}$. For the monodisperse packing this can be calculated analytically while for the polydisperse packings the normal vector components are extracted from the simulation when the system is at equilibrium.

From continuum theory, the (square of the) P-wave speed in z-direction is:

$$
\mathrm{v}_{p z}^{2}=C_{z z z z} / \rho
$$

with the material mass-density $\rho\left(=2000 \mathrm{~kg} / \mathrm{m}^{3}\right)$; these results are given in the row labeled "theory" of table 1 .

This approach implies however the assumption of a constant, time-invariant material tensor, which can hold only for small deformations, and does not allow for opening or closing of contacts or even large scale rearrangements. Furthermore, the large wavelength limit is implied. This is all verified for the mono-disperse packing, and explains the quite good agreement between theory and simulations, where the discrepancy is only about 3\% (see table 1, column ' 0 '). As soon as polydispersity is introduced, and already for small values, the material tensor is no longer time-invariant and opening and closing of contacts is observed. Indeed in the case $(\Delta a)=2 \delta$ the discrepancy between theory and simulation rises up to $8.5 \%$ (see table 1 , column ' $2 \delta$ '). Although a linear contact law drives the interaction between the particles, the opening and closing of contacts introduces a non-linear interaction, and thus makes the linear theoretical approach inappropriate. A more suitable theory should not only rely on the static initial configuration of the system but should take into account the dynamic nature of the phenomenon as well, in order to determine a realistic effective stiffness for the material. For a different approach on the moduli, that considers random arrays of particles, see Ref. [4]. 


\section{C.2. Wave frequency contents}

As one can see from Fig. 3, the signal is strongly affected for $(\Delta a)=2 \delta$ as compared to the monodisperse case, $(\Delta a)=0$, see Ref. [5] for more details on monodisperse packings. Qualitatively the first peak is shifted to the right, which is explained by a lower propagation speed (see section C.1) due to the smaller coordination number. The signal amplitude is smaller, which indicates that some energy has been transferred in other types of motion (waves of different nature, shear wave for example, and/or with different propagation direction). Also the coda of the signal that runs through a polydisperse packing is clearly irregular, while it is periodic in the monodisperse case.
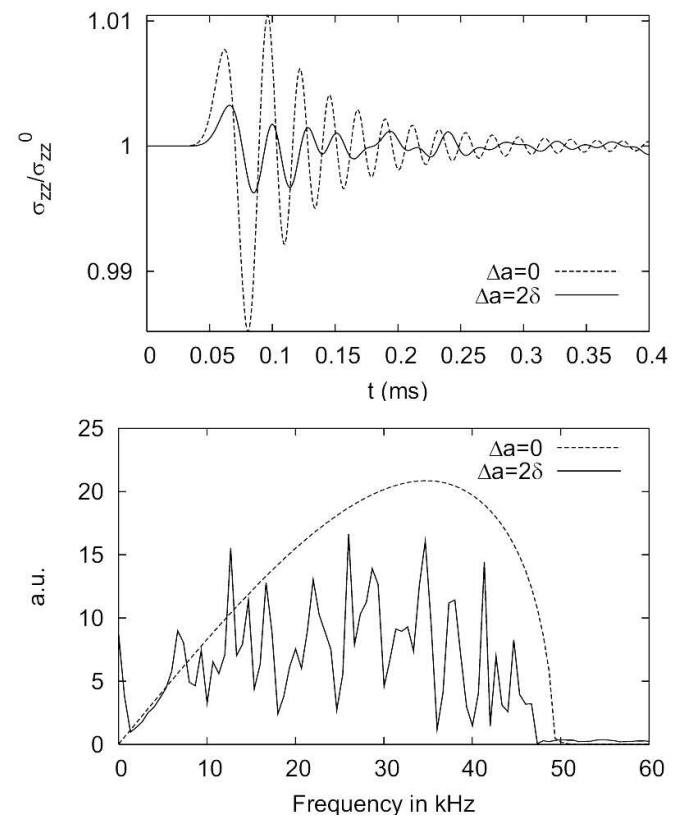

Fig. 3. $\underline{\text { Top: }}$ : Normal stress $\left(\sigma_{\mathrm{zz}}\right)$ scaled by the equilibrium stress $\left(\sigma_{\mathrm{zz}}\right)$ as function of time at a distance of 10 layers from the source for the monodisperse $(\Delta a=0)$ and a polydisperse $(\Delta a=2 \delta)$ system. Bottom: Fourier power spectrum of the two stress-time signals.

In order to analyse in more detail this observation, we examine the frequency content by first calculating the Fourier spectrum in time, see Fig. 3 (Bottom). Due to the irregularities present at the contact level, some frequencies seem to be filtered. The spectrum plotted against the position in z-direction in Fig. 4, shows the same features with intensity increasing with distance from the source as discussed in the following. The frequencyspace diagrams for the polydisperse packings with $(\Delta a)=\delta / 2,(\Delta a)=\delta$, and $(\Delta a)=2 \delta$ are here obtained from simulations with friction active. This shows the evolution of the frequency content of the stress-time signals when the wave propagates away from the source. For the first case (Fig. 4, top, $(\Delta a)=\delta / 2$ ) the power spectrum does not change much while the wave is propagating through the system, the bending observed starting at about 50 layers from the source is only due to the limited time window of the collected data. The same diagram for the monodisperse case does not offer visual differences with this case and is therefore not shown here. However, for the polydisperse frictionless case, $(\Delta a)=2 \delta$ (Fig. 4, center), the frequency range is strongly reduced as the wave propagates away from the source, with a clearly persistent low frequency transmission range around 8 $\mathrm{kHz}$. Also some frequencies seem to last longer than others as around $35 \mathrm{kHz}$ for example. (Note that in the case $(\Delta a)=\delta$ (data not shown), the same observation is made also around $35 \mathrm{kHz})$. In the last case, $(\Delta a)=2 \delta$, with friction (Fig. 4, bottom), the frequency spectrum extends to higher frequencies as a consequence of the tangential spring at the contact, which induces a higher particle "eigenfrequency" and thus a larger bulk stiffness. The frequency range of the wave is limited earlier than in the frictionless case (some special frequencies propagate further) and the remaining low frequency band is broader and centered at about $10 \mathrm{kHz}$.
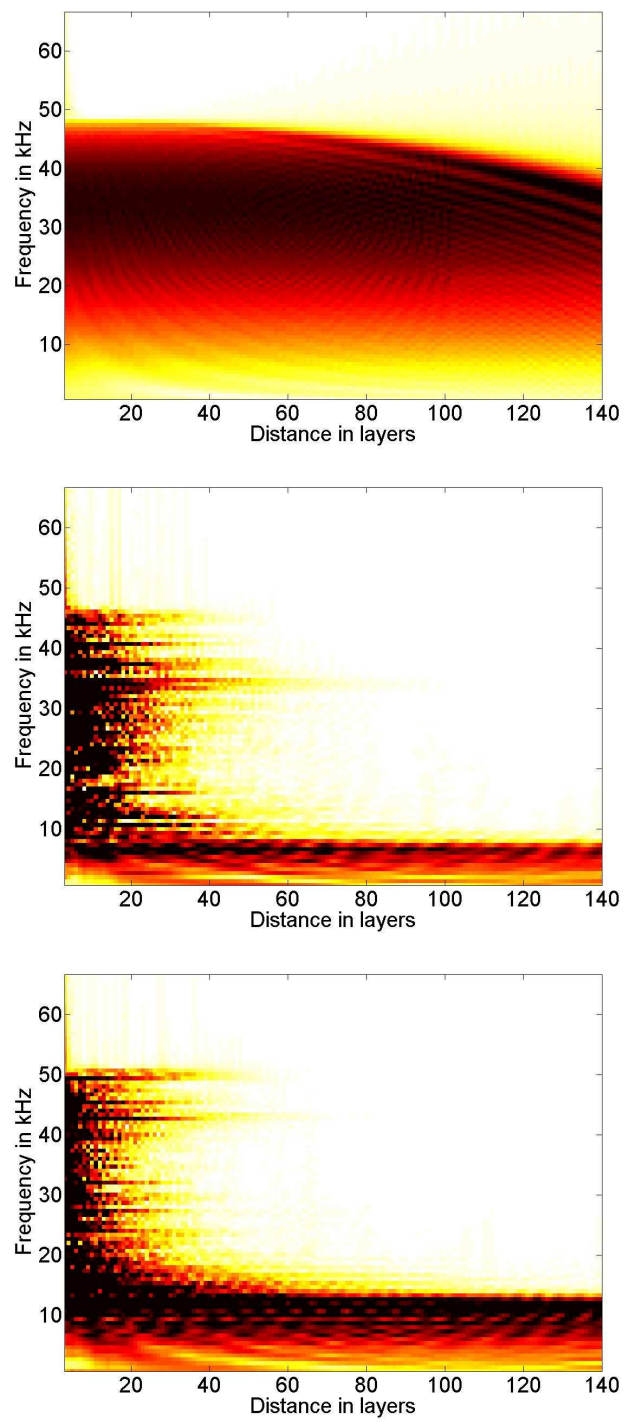

Fig. 4. Frequency-space diagram (darkness correspond to the amplitude, absolute value, of the Fourier coefficients, black is high and white is low) for the P-wave propagating in $\mathrm{z}$-direction for three different polydisperse packings (from top to bottom: $(\Delta a)=\delta / 2$, and $(\Delta a)=2 \delta$ without and with friction, respectively). 
Next, the two-dimensional Fourier transform in time and space has been calculated for the P-wave travelling through the system. The dispersion relation (frequency versus wave number) obtained for the monodisperse and the polydisperse (case $(\Delta a)=\delta / 2$ ) packing is a sinus (see Fig. 5, top), visually identical to the monodisperse case. This has been studied in more detail in Ref. [5,6] for a different excitation method but showing similar behaviour.
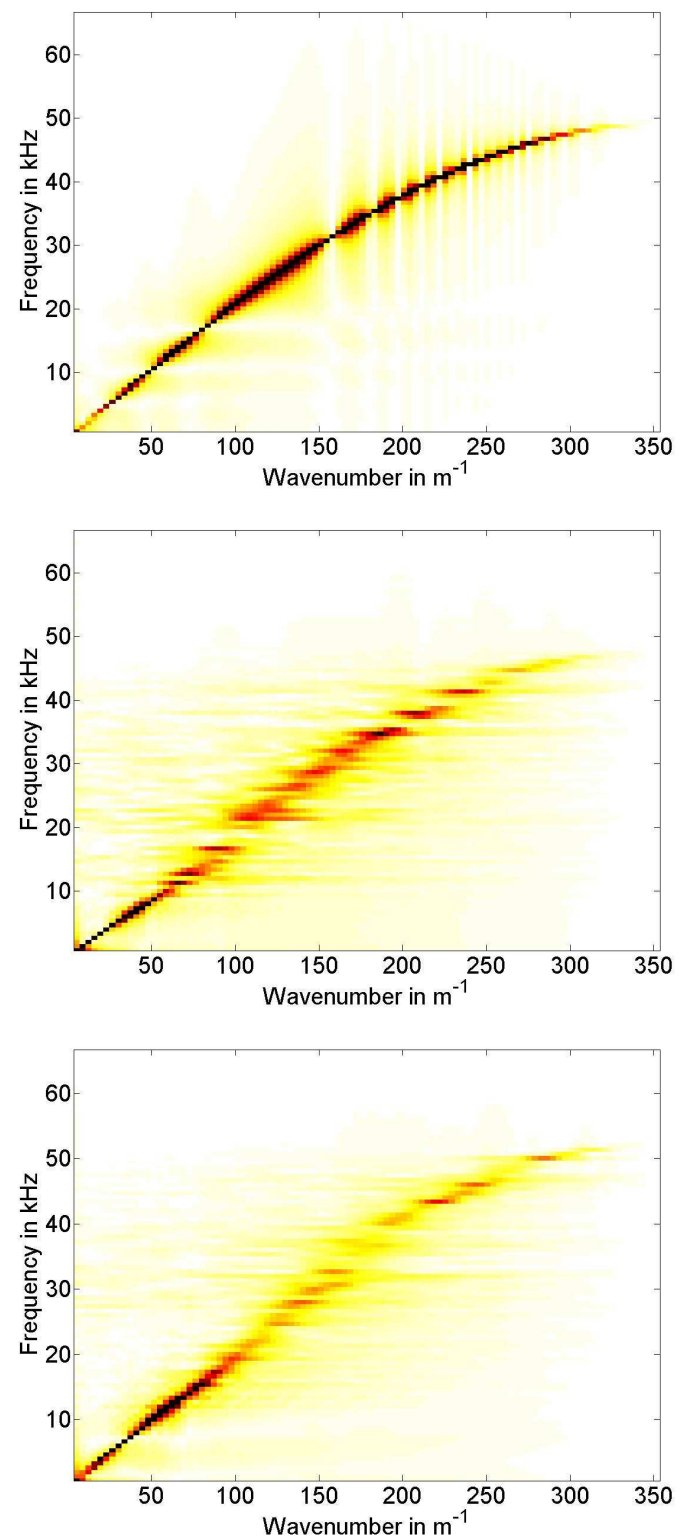

Fig. 5. Dispersion relation (darkness correspond to the amplitude, absolute value, of the Fourier coefficients, black is high and white is low) for the same data as in Fig. 4.

For the two polydisperse packings, $(\Delta a)=2 \delta$, without and with friction, the dispersion relations obtained (see Fig. 5) are much broader, more noisy, and random gaps seem to appear, although the sine shape is still visible. In the "low frequency/large wavelength" regime a clear linear behaviour (with a corresponding wave speed) is evidenced. The presence of friction seems to extend or stabilize this regime. For the non-linear, non-propagating regime, a weaker signal is obtained in the frictional case, as in addition to the geometrical spreading, the presence of friction accentuate the compressive- to shear-mode conversion and activates also the rotational modes [5].

\section{Conclusions}

Wave propagation was examined in threedimensional regular (crystal) monodisperse and slightly polydisperse packings of spheres with and without friction, for compressive $(\mathrm{P})$ propagation modes.

For the wave speed, quantitative agreement has been obtained between simulations and theoretical predictions based on a micro-macro computation of the stiffness material tensor for the monodisperse packing in the large wavelength limit. However, the quality of agreement decays when the polydispersity is increasing. The opening and closing of contacts already present for small perturbations due to tiny size variations creates non-linear effects.

Finally an interesting filtering of most higher frequencies is observed as polydispersity is increasing. Due to polydispersity, the dispersion relation becomes noisier while maintaining its sine profile approximately. The influence of friction could be characterised in so far, that friction increases the stiffness of the sample and thus the available frequency range, but also seems to stabilize the "linear" long wavelength regime in the dispersion relation.

\section{E. Acknowledgements}

This work is part of the research program of the "Stichting voor Fundamenteel Onderzoek der Materie" (FOM), financially supported by the "Nederlandse Organisatie voor Wetenschappelijk Onderzoek" (NWO) and the Stichting Shell Research.

\section{F. Literature}

[1] E. Somfai, J.-N. Roux, J. H. Snoeijer, M. van Hecke, and W. van Saarloos: Elastic wave propagation in confined granular systems, Phys. Rev. E 72(2), 021301 (2005).

[2] S. Luding: Stress distribution in static two-dimensional granular model media in the absence of friction, Phys. Rev. E 55, 4720-4729 (1997).

[3] S. Luding: Micro-macro transition for anisotropic, frictional granular packings, Int. J. Sol. Struct. 41, 58215836 (2004).

[4] J. T. Jenkins et al: Fluctuations and the effective moduli of an isotropic, random aggregate of identical, frictionless spheres, Journal of the Mechanics and Physics of Solids, 53(1), 197-225 (2004).

[5] O. Mouraille, W. A. Mulder and S. Luding: Sound wave acceleration in granular materials, J. Stat. Mech. P07023 (2006).

[6] O. Mouraille and S. Luding, Mechanic waves in sand: Effect of polydispersity, PARTEC 2007, Nuremberg, Germany, CD proceedings. 